Purdue University

Purdue e-Pubs

$1-1-1980$

\title{
Maximum Likelihood Estimation of Label Imperfection Probabilities and Its Use in the Identification of Mislabeled Patterns
}

\author{
C. B. Chittineni
}

Follow this and additional works at: http://docs.lib.purdue.edu/lars_symp

Chittineni, C. B., "Maximum Likelihood Estimation of Label Imperfection Probabilities and Its Use in the Identification of Mislabeled Patterns" (1980). LARS Symposia. Paper 352.

http://docs.lib.purdue.edu/lars_symp/352

This document has been made available through Purdue e-Pubs, a service of the Purdue University Libraries. Please contact epubs@purdue.edu for additional information. 
Reprinted from

Symposium on

Machine Processing of

Remotely Sensed Data

and

\author{
Soil Information Systems
}

and

\title{
Remote Sensing and Soil Survey
}

\author{
June 3-6, 1980 \\ Proceedings
}

The Laboratory for Applications of Remote Sensing

\author{
Purdue University \\ West Lafayette \\ Indiana 47907 USA \\ IEEE Catalog No. \\ 80CH1533-9 MPRSD \\ Copyright (C) 1980 IEEE
}

The Institute of Electrical and Electronics Engineers, Inc.

Copyright (C) 2004 IEEE. This material is provided with permission of the IEEE. Such permission of the IEEE does not in any way imply IEEE endorsement of any of the products or services of the Purdue Research Foundation/University. Internal or personal use of this material is permitted. However, permission to reprint/republish this material for advertising or promotional purposes or for creating new collective works for resale or redistribution must be obtained from the IEEE by writing to pubs-permissions@ieee.org.

By choosing to view this document, you agree to all provisions of the copyright laws protecting it. 
MAXIMUM LIKELIHOOD ESTIMATION OF LABEL IMPERFECTION PROBABILITIES AND ITS USE IN THE IDENTIFICATION OF MISLABELED PATTERNS

\section{C.B. CHITTINENI}

Lockheed Engineering and Management Services Company, Inc.

\section{ABSTRACT}

This paper presents the problem of estimating label imperfections and the use of the estimation in identifying mislabeled patterns. Expressions for the maximum likelihood estimates of classification errors and a priori probabilities are derived from the classification of a set of labeled and unlabeled patterns. Expressions also are presented for the asymptotic variances of probability of correct classification and proportions. Simple models are developed for imperfections in the labels and for classification errors and are used in the formulation of a maximum likelihood estimation scheme. Schemes are presented for the identification of mislabeled patterns in terms of thresholds on the discriminant functions for both two-class and multiclass cases. Expressions are derived for the probability that the imperfect label identification scheme will result in a wrong decision and are used in computing thresholds. Furthermore, the results of practical applications of these techniques in the processing of remotely sensed multispectral data are presented.

\section{INTRODUCTION}

In the practical applications of pattern recognition (such as in the processing of remotely sensed imagery data), obtaining labels is a difficult problem. Acquiring labels is expensive, and very often these labels are imperfect.

Several scientists have investigated the problem of pattern recognition with imperfectly labeled patterns.1-7 Kashyap proposed an. iterative training procedure for a two-class case. Shanmugam and Breiphol ${ }^{3}$ developed an errorcorrecting procedure for disjoint densities using Parzen estimators. Chittineni $i^{4} 7$ investigated the problem of learning with imperfectly labeled patterns and studied the applicability of probabi-

*Under Contract NAS 9-15800 at the National Aeronautics and Space Administration, Lyndon B. Johnson Space Center, Houston, Texas 77058. listic distance measures for feature selection with imperfectly labeled patterns. Most of these proposed schemes require the knowledge of probabilities of label imperfections, which usually are not available.

Several authors considered the problem of estimating recognition system performance. $8^{-13}$ Highleyman ${ }^{8}$ investigated the problem of estimating the probability of error of a given classifier both for known and unknown a priori probabilities. Fukunaga and Kessel $1^{9}$ examined the problem of estimating the probability of error from unclassified samples. Havens et al.10 reported the experimental results of estimating the probability of error from unclassified samples using remotely sensed agricultural data. Chowl i established a relationship between error and rejection rates which is useful in estimating the probability of error from unclassified samples.

In practice, the situation often arises in which a set of imperfectly labeled test patterns and a set of unlabeled patterns are available. (For example, in remote sensing, a set of labeled patterns called type 2 dots and a set of unlabeled patterns are usually available.) This paper presents the problem of estimating recognition system performance and probabilities of label imperfections as maximum likelihood estimates from the classifier decisions of labeled and unlabeled patterns. The probabilities of the estimated label imperfections are then used in developing schemes for the identification of mislabeled patterns. The paper is organized in the following manner.

Assuming no imperfections in the labels, expressions are derived for the maximum likelihood estimates of probability of error, probability of correct classification, and a priori probabilities (section II); also, in this section, expressions are derived for the asymptotic variances of probability of correct classification and a priori probabilities. In section III, imperfections in the labels are introduced, models for the label imperfections and probabilities of errors are developed, and the simulation results from the processing of remotely sensed data are presented. Methods of identifying mislabeled patterns for

CH1533-9/80/0000-0158 \$00.75 (C) 1980 IEEE

1980 Machine Processing of Remotely Sensed Data Symposium 
both two-class and multiclass cases are reported in section IV, and the results of their applications in processing remotely sensed data are described. Conclusions are presented in section $V$.

II. MAXIMUM LIKELIHOOD ESTIMATION OF PROBABILITY OF ERROR, PROBABILITY OF CORRECT CLASSIFI -

CATION, AND A PRIORI PROBABILITIES

In this section, expressions are derived for the maximum likelihood estimates of probability of error, probability of correct classification, and proportions. Also, expressions for the asymptotic variance of probability of correct classification and proportion estimates are derived. It is assumed that the classifier is designed and the classifier classifications of a set of labeled and unlabeled patterns are obtained. [In a situation involving remote sensing, the labeled patterns are the test set or type 2 dots and the unlabeled patterns are the spectral values of the picture elements (pixels) for which no labels are available.] In this section, the labels of the test patterns are assumed perfect; in section III, the labels are assumed to be imperfect. The classifier classifications of the labeled and unlabeled sets are illustrated in table 1.

Let $\omega$ be the given label and $\omega_{c}$ be the classifier label. Let $\lambda_{i j}=P\left(\omega=i \mid \omega_{c}=j\right)$ be the probability that the true label is $i$, given that the classifier label is $j$. Let $p_{i j}=P(\omega=i$, $\omega_{c}=j$ ) be the probability that the true label of the pattern is $i$ and the classifier label is $j$. Let $P_{c}(i)=P\left(w_{c}=i\right)$ be the probability that the classifier classifies a pattern into class $i$ and $P_{j}=P(\omega=i)$ be the a priori probability of class $i$. Then we obtain

$$
\begin{aligned}
P_{i j} & =P\left(\omega=i, \omega_{c}=j\right) \\
& =P\left(\omega_{c}=j\right) P\left(\omega=i \mid \omega_{c}=j\right) \\
& =P_{c}(j) \lambda_{i j}
\end{aligned}
$$

Since each classification is independent, the likelihood function of the observed $\mathrm{m}^{\prime} \mathrm{s}$ and $X^{\prime} \mathrm{s}$ can be written as

$$
\begin{aligned}
L & =c \prod_{i=1}^{M} \prod_{j=1}^{M}\left(p_{i j}\right)^{m_{i j}} \prod_{j=1}^{M}\left[p_{c}(j)\right]^{x_{j}} \\
& =c \prod_{i=1}^{M} \prod_{j=1}^{M}\left(\lambda_{i j}\right)^{m_{i j}} \prod_{j=1}^{M}\left[p_{c}(j)\right]^{x_{j}+m} \cdot j
\end{aligned}
$$

where $C$ is a constant. The constraints on $\lambda_{i j}$ and $P_{c}(j)$ are

$$
\left.\begin{array}{c}
\sum_{j=1}^{M} \lambda_{i j}=1 ; j=1,2, \cdots, M \\
\sum_{j=1}^{M} P_{c}(j)=1
\end{array}\right\}
$$

\begin{tabular}{|c|c|c|c|c|c|}
\hline \multirow{2}{*}{ True label } & \multicolumn{4}{|c|}{ Classifier label } & \multirow{2}{*}{$\begin{array}{l}\text { Number belonging } \\
\text { to each class }\end{array}$} \\
\hline & 1 & 2 & $\cdots$ & M & \\
\hline 1 & $m_{11}$ & $m_{12}$ & $\cdots$ & $m_{1 M}$ & $\mathrm{~m}_{1}$. \\
\hline 2 & $m_{21}$ & $m_{22}$ & $\cdots$ & $m_{2 M}$ & $m_{2}$. \\
\hline$\vdots$ & $\vdots$ & $\vdots$ & & $\vdots$ & $\vdots$ \\
\hline M & $m_{M 3}$ & $m_{12}$ & $\cdots$ & $T_{\text {man }}$ & $m_{M}$. \\
\hline $\begin{array}{l}\text { Number classified } \\
\text { into each class }\end{array}$ & $m .1$ & $\mathrm{~m} .2$ & $\cdots$ & ${ }^{m} \cdot M$ & $m=m$. \\
\hline
\end{tabular}

Table 1. Classifications of Labeled and Unlabeled Sets

(a) Confusion matrix of labeled test set

(b) Matrix of classifications of unlabeled set

\begin{tabular}{|c|c|c|c|}
\hline \multicolumn{4}{|c|}{ Classifier label } \\
\hline 1 & 2 & $\cdots$ & $M$ \\
\hline$x_{1}$ & $x_{2}$ & $\cdots$ & $x_{M}$ \\
\hline
\end{tabular}

where

$m_{i j} \quad=$ number of labeled patterns for which the true or given label is $i$ and the classifier label is $j$

$M \quad=$ number of classes

$m_{i} . \quad=\sum_{j=1}^{M} m_{i j}$

$m_{. j} \quad=\sum_{i=1}^{M} m_{i j}$

$m=m_{.}=\sum_{i=1}^{M} \sum_{j=1}^{M} m_{i j}$, the total number of labeled patterns

$x_{j} \quad=$ number of uniabeled patterns for which the classifier label is

The objective is to find the values for $\lambda_{i j}$ and $P_{c}(j)$ which maximize $L$, subject to the constraints of equation (3). Since the logarithm is a monotonic function of its argument, taking the logarithm of $L$ and introducing Lagrangian multipliers yields

$$
\begin{aligned}
L^{\prime}= & \log c+\sum_{j=1}^{M} \sum_{j=1}^{M} m_{i j} \log \left(\lambda_{i j}\right)+\sum_{j=1}^{M}\left(x_{j}+m_{. j}\right) \log \left[p_{c}(j)\right] \\
& +\sum_{j=1}^{M} r_{j}\left(\sum_{i=1}^{M} \lambda_{i j}-1\right)+s\left[\sum_{j=1}^{M} P_{c}(j)-1\right]
\end{aligned}
$$

where $r_{j}(j=1,2, \cdots, M)$ and $s$ are Lagrangian multipliers. Differentiating $L$ ' with respect to $P_{C}(j)$ and $s$, equating the resulting expressions to zero, and solving for $P_{c}(j)$ results in

$$
\hat{p}_{c}(j)=\frac{m_{\cdot j}+x_{j}}{\sum_{l=1}^{M}\left(m_{\cdot l}+x_{l}\right)}
$$


Similarly, the maximum likelihood estimate of $\lambda_{i j}$ can be obtained as

$$
\hat{\lambda}_{i j}=\frac{m_{i j}}{m_{. j}}
$$

From the invariance property of the maximum likelihood estimators, the maximum likelihood estimate $\hat{P}_{C c}$ for the probability of correct classification $P_{C C}$ can be obtained from the expression

$$
\begin{aligned}
P_{c c} & =\sum_{i=1}^{M} P\left(\omega=i, \omega_{c}=i\right) \\
& =\sum_{i=1}^{M} P_{c}(i) \lambda_{i j}
\end{aligned}
$$

Using equations (5) and (6) in equation (7) yields

$$
\hat{p}_{c c}=\frac{\sum_{i=1}^{M} \frac{m_{i j}}{m_{. i}}\left(m_{. i}+x_{i}\right)}{\sum_{\ell=1}^{M}\left(m_{. \ell}+x_{\ell}\right)}
$$

An intuitive justification for $\hat{\mathrm{P}}_{\mathrm{Cc}}$ may be given as follows. The ratio $\left(m_{i j} \mid m_{i}\right)$ gives the proportion of the patterns truly belonging to proportion of the patterns classified into class $i$. Multiplying this ratio by $\left(m+i+x_{j}\right)$ and summing Multiplying to $M$ gives an estimate for the number of it from 1 to $M$ classified patterns from all patterns in correctlysified classes. The estimate of $P_{c c}$ is the classified classes. then divided by the proportion $P_{i}$ may be obtained estimate $i$

$$
\begin{aligned}
P_{i} & =P(\omega=i) \\
& =\sum_{j=1}^{M} P\left(\omega=1, \omega_{c}=j\right) \\
& =\sum_{j=1}^{M} P_{c}(j) \lambda_{i j}
\end{aligned}
$$

From equations (5), (6), and (9), the following is obtained.

$$
\hat{p}_{i}=\frac{\sum_{j=1}^{M}\left[\frac{m_{i j}}{m_{\cdot j}}\left(m_{\cdot j}+x_{j}\right)\right]}{\sum_{l=1}^{M}\left(m_{\cdot l}+x_{l}\right)}
$$

Different probabilities of error can be written as

$$
P\left(\omega_{c}=j \mid \omega=1\right)=\frac{P\left(\omega_{c}=j\right) P\left(\omega=i / \omega_{c}=j\right)}{P(\omega=1)}
$$

Using equations (5), (6), and (10) in equation (11) obtains the maximum likelihood estimates $\left[\hat{P}\left(\omega_{c}=j \mid \omega=j\right)\right]$ for different probabilities of error.

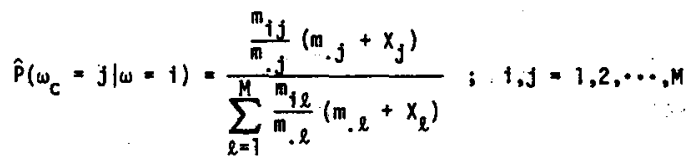

The estimate of equation (12) can be interpreted as follows. It is the ratio of the number of patterns that truly belong to class i but were classified into class $j$ to the total number of patterns that truly belong to class $i$ from the patterns classified into a11 classes.

In the following, expressions are derived for the asymptotic variance of the estimates of the probability of correct classification and proportions: From equation (7), the estimated $\hat{P}_{\mathrm{C} c}$ can be written as

$$
\hat{p}_{c c}=\sum_{i=1}^{M} \hat{p}_{c}(i) \hat{\lambda}_{i j}
$$

The delta method ${ }^{14}$ is used to compute the asymptotic variance of $\hat{P}_{\mathrm{Cc}}$. This involves expanding $\beta_{C C}$ in a Taylor series around the true value $P_{C c}=\sum_{i=1}^{M} P_{c}(i) \lambda_{i j}$. The result of this expansion

$$
\begin{aligned}
\operatorname{Var}\left(\hat{\mathrm{P}}_{c c}\right)= & \sum_{i=1}^{M} \sum_{j=1}^{M} \operatorname{cov}\left(\hat{\lambda}_{i j} \hat{\lambda}_{j j}\right) \frac{\partial P_{c c}}{\partial \lambda_{i j}} \frac{\partial P_{c c}}{\partial \lambda_{j j}} \\
& +\sum_{i=1}^{M} \sum_{j=1}^{M} \operatorname{cov}\left[\hat{\lambda}_{i j} \hat{P}_{c}(j)\right] \frac{\partial P_{c c}}{\partial \lambda_{j i}} \frac{\partial P_{c c}}{\partial P_{c}(j)} \\
& +\sum_{j=1}^{M} \sum_{j=1}^{M} \operatorname{cov}\left[\hat{\beta}_{c}(i) \hat{\lambda}_{j j}\right] \frac{\partial P_{c c}}{\partial P_{c}(j)} \frac{\partial P_{c c}}{\partial \lambda_{j j}} \\
& +\sum_{i=1}^{M} \sum_{j=1}^{M} \operatorname{cov}\left[\hat{p}_{c}(j) \hat{P}_{c}(j)\right] \frac{\partial P_{c c}}{\partial P_{c}(j)} \frac{\partial P_{c c}}{\partial P_{c}(j)}
\end{aligned}
$$

The number of independent parameters is $2 M-1$; name $1 y, \lambda 11, \lambda 22, \cdots, \lambda M M$ and $P_{C}(1), P_{c}(2)$, $\cdots, P_{c}(M-1)$. If these parameters are labeled by $\delta_{i}, i c=1,2, \cdots, 2 M-1$, the $(2 M-1)$ by $(2 M-1)$ information matrix, the general term of which is given by $E\left(-\frac{\partial^{2} \log L}{\partial \delta_{j} \partial \delta_{j}}\right)$, can be evaluated from equation (2). Carrying out these calculations and inverting the resulting matrix yields the variance-covariance matrix of $\lambda_{i j}, \mathbf{i}=1,2, \cdots, M$, and $P_{c}(j), j=1,2, \cdots, M-1$. From this, the following are obtained.

$$
\begin{aligned}
& \operatorname{Var}\left[\hat{P}_{c}(i)\right]=\frac{P_{c}(i)\left[1-P_{c}(i)\right]}{N} \\
& \operatorname{Cov}\left[\hat{P}_{c}(i) \hat{P}_{c}(j)\right]=-\frac{P_{c}(i) P_{c}(j)}{N} \\
& \operatorname{Var}\left(\hat{\lambda}_{i j}\right)=\frac{\lambda_{i j}\left(1-\lambda_{1 i}\right)}{m P_{c}(i)}
\end{aligned}
$$




$$
\operatorname{Cov}\left[\hat{\lambda}_{i j} \hat{p}_{c}(j)\right]=\operatorname{Cov}\left[\hat{P}_{c}(i) \hat{\lambda}_{j j}\right]=\operatorname{Cov}\left(\hat{\lambda}_{i j} \hat{\lambda}_{k k}\right)=0
$$

for all $i$ and $j, i \neq k$, where

$$
N=\sum_{\ell=1}^{M} x_{\ell}
$$

Substituting equations (5) through (19) into equation (14) yields an expression for the $\operatorname{Var}\left(\hat{P}_{\mathrm{CC}}\right)$ as follows.

$$
\begin{aligned}
\operatorname{Var}\left(\hat{P}_{c c}\right)= & \sum_{i=1}^{M} \frac{\lambda_{i j}\left(1-\lambda_{i j}\right)}{m P_{c}(i)} p_{c}^{2}(i)+\sum_{i=1}^{M} \sum_{\substack{j=1 \\
j \neq 1}}^{M} \frac{\left[-p_{c}(i) P_{c}(j)\right]}{N} \lambda_{i j} \lambda_{j j} \\
& +\sum_{i=1}^{M} \frac{P_{c}(i)\left[1-P_{c}(i)\right]}{N} \lambda_{i i}^{2} \\
& =\sum_{i=1}^{M} \frac{\lambda_{j i}\left(1-\lambda_{i j}\right) P_{c}(1)}{m}+\frac{\left[\sum_{i=1}^{M} P_{c}(i) \lambda_{i j}^{2}-p_{c c}^{2}\right]}{N}
\end{aligned}
$$

Following a similar analysis, an expression may be obtained for the asymptotic variance of the a priori probability estimator. 15

$\operatorname{Var}\left(\hat{A}_{i}\right)=\sum_{j=1}^{M} \frac{\lambda_{i j}\left(1-\lambda_{i j}\right) P_{c}(j)}{m}+\frac{\left[\sum_{j=1}^{M} P_{c}(j) \lambda_{i j}^{2}-P_{c}^{2}(i)\right]}{N}$

In general, one can obtain expressions for sample sizes $m$ and $N$, either by minimizing the $\operatorname{Var}\left(\hat{P}_{C C}\right)$ or by minimizing the $\operatorname{Var}\left(\hat{P}_{i}\right)$, subject to some cost constraints.

\section{MAXIMUM LIKELIHOOD ESTIMATION WITH LABEL IMPERFECTIONS}

In practical situations, obtaining labels is expensive, and very often these labels are imperfect. In this section, we formulate the problem of estimating, with imperfections in the labels, the various quantities considered in section II.

It is assumed that the classifier is trained on representative data, and a set of labeled patterns (possibly with imperfect labels) and a set of uniabeled patterns are presented to the classifier. The classifier classifies these patterns, and the results are matrices similar to table 1 . Now the various quantities are defined as follows.

Let $\omega^{\prime}$ be the imperfect label, $P_{j}^{\prime}=P\left(\omega^{\prime}=i\right)$ be the a priori probability that the imperfect label is $i, p_{i j}^{\prime}=P\left(\omega^{\prime}=j, \omega_{c}=j\right)$ be the probability that the imperfect label is $i$, and $j$ be the classifier label. Consider

$$
\begin{aligned}
P_{i j}^{\prime} & =P\left(\omega^{\prime}=i, \omega_{c}=j\right) \\
& =\sum_{\ell=1}^{M} P\left(\omega^{\prime}=1, \omega=\ell, \omega_{c}=j\right)
\end{aligned}
$$

$$
\begin{aligned}
& =\sum_{\ell=1}^{M} P\left(\omega^{\prime}=i \mid \omega=\ell, \omega_{c}=j\right) P\left(\omega=\ell, \omega_{c}=j\right) \\
& =\sum_{\ell=1}^{M} P\left(\omega^{\prime}=j \mid \omega=\ell\right) P\left(\omega_{c}=j \mid \omega=\ell\right) P(\omega=\ell)
\end{aligned}
$$

where it is assumed that

$$
P\left(\omega^{\prime}=i \mid \omega=\ell\right)=P\left(\omega^{\prime}=\left.i\right|_{\omega}=\ell_{o} \omega_{c}=j\right)
$$

This assumption states that, given the true label and the classifier label, the imperfect label depends only on the true label. This is a reasonable assumption. In acquiring the label for a pattern, the labeler depends heavily on the true label of the pattern and virtually does not know the classifier label. (In labeling a pixel in imagery data, the assigned label depends on the true label of the pixel, and its neighbors and on some other data such as ancillary information.) Now consider

$$
\begin{aligned}
P_{c}(j) & =P\left(\omega_{c}=j\right) \\
& =\sum_{\ell=1}^{M} P\left(\omega_{c}=j, \omega=\ell\right) \\
& =\sum_{\ell=1}^{M} P\left(\omega_{c}=j \mid \omega=\ell\right) P(\omega=\ell)
\end{aligned}
$$

Substituting equations (22) and (24) into the likelihood function and taking the logarithm results in

$$
\begin{aligned}
L= & \log C+\sum_{i=1}^{M} \sum_{j=1}^{M} m_{j j} \log \left[\sum_{\ell=1}^{M} P\left(\omega^{\prime}=i \mid \omega=\ell\right) P\left(\omega_{c}=j \mid \omega=\ell\right) P(\omega=\ell)\right] \\
& +\sum_{j=1}^{M} x_{j} \log \left[\sum_{\ell=1}^{M} P\left(\omega_{c}=j \mid \omega=\ell\right) P(\omega=\ell)\right]
\end{aligned}
$$

Finding closed-form solutions for the parameters by maximizing $L$ seems to be difficult, since the resulting equations become coupled in terms of parameters. However, opt imization techniques, such as the Davidon-Fletcher-Powell procedure, can be used to maximize L.16-18 Now, the problem can be formulated as

Find: $P\left(\omega^{\prime}=1 \mid \omega=\ell\right), P\left(\omega_{c}=j \mid \omega=\ell\right), P(\omega=\ell) ; 1, j, \ell=1,2, \cdots, M$

such that $L$ is maximized subject to the following constraints.

$$
\begin{gathered}
\sum_{j=1}^{M} P\left(\omega^{\prime}=1 \mid \omega=\ell\right)=1 ; \ell=1,2, \ldots, M \\
\sum_{j=1}^{M} P\left(\omega_{c}=j \mid \omega=\ell\right)=1 ; \ell=1,2, \cdots, M \\
\sum_{\ell=1}^{M} P(\omega=\ell)=1 \\
P\left(\omega^{\prime}=1 \mid \omega=\ell\right) \geq 0 ; i, \ell=1,2, \cdots, M \\
P\left(\omega_{C}=j \mid \omega=\ell\right) \geq 0 ; j, \ell=1,2, \ldots, M \\
P(\omega=\ell) \geq 0 ; \ell=1,2, \cdots, M
\end{gathered}
$$


The numbers of parameters, equality constraints, and inequality constraints as a function of $M$ are $\left(2 M^{2}+M\right),(2 M+1)$, and $\left(2 M^{2}+M\right)$, respectively. The numbers of parameters and constraints increase with the square of the number of classes, resulting in a large number of degrees of freedom for the optimization problem. However, the numbers of constraints and parameters can be reduced by modeling the label imperfections and the probabilities of misclassification.

\section{A. MAXIMUM LIKELIHOOD ESTIMATION WITH SIMPLIFIED MODELS}

This section provides (1) models for label imperfections and probabilities of misclassification and (2) a formulation of the problem of maximum likelihood estimation. To develop a model for describing the probabilities of imperfections in the labels, consider the following.

1. If there are no imperfections in the labels, for different $i$ and $j$,

$$
\text { and } \left.\quad \begin{array}{l}
P\left(\omega^{\prime}=i \mid \omega=1\right)=1 \\
P\left(\omega^{\prime}=j \mid \omega=1\right)=0
\end{array}\right\}
$$

2. If the imperfect label for a pattern is assigned purely at random, irrespective of its true label, for different $i$ and $j$,

and

$$
\left.\begin{array}{l}
P\left(\omega^{\prime}=i \mid \omega=i\right)=\frac{1}{M} \\
P\left(\omega^{\prime}=j \mid \omega=i\right)=\frac{1}{M}
\end{array}\right\}
$$

Since in a practical situation, the assignment of a label lies somewhere between the above two extremes, the imperfections in the labels can be modeled through a parameter $\theta_{1}$, which lies between 0 and 1 as

$$
\left.\begin{array}{c}
P\left(\omega^{\prime}=1 \mid \omega=1\right)=\frac{\left(1-\theta_{1}\right)}{M}+\theta_{1} \\
P\left(\omega^{\prime}=j \mid \omega=i\right)=\frac{\left(1-\theta_{1}\right)}{M}
\end{array}\right\}
$$

where $0 \leq \theta_{1} \leq 1$.

From equations (27) through (29), it is easily seen that $\theta_{1}=1$ denotes no imperfections in the labels and $\theta_{1}=0$ denotes random labeling. For the model of equation. (29), it can be shown that

$$
\sum_{j=1}^{M} P\left(\omega^{\prime}-j \mid \omega=1\right)=1
$$

thus satisfying the probability rule. Similarly, classification errors can be modeled as follows.

1. If there are no classification errors, for different $i$ and $j$, and

$$
\left.\begin{array}{l}
P\left(\omega_{c}=1 \omega_{\omega}=1\right)=1 \\
P\left(\omega_{c}=j \omega_{\omega}=1\right)=0
\end{array}\right\}
$$

2. If the classifier is making random decisions, for different $i$ and $j$,

and

$$
\left.\begin{array}{l}
P\left(\omega_{c}=\left.1\right|_{\omega}=1\right)=\frac{1}{M} \\
P\left(\omega_{c}=j / \omega=1\right)=\frac{1}{M}
\end{array}\right\}
$$

Since, in general, the truth lies somewhere between the above two extremes, the classification errors can be modeled through a parameter $\theta_{2}$, which lies between 0 and 1 as

and

$$
\left.\begin{array}{c}
P\left(\omega_{c}=f \mid \omega=1\right)=\frac{\left(1-\theta_{2}\right)}{M}+\theta_{2} \\
P\left(\omega_{c}=j \mid \omega=1\right)=\frac{\left(1-\theta_{2}\right)}{M}
\end{array}\right\}
$$

where $0<\theta_{2}<1$. As before, it can be seen that this modēl satisfies the postulates of probability.

Let $\lambda_{1}=\left(1-\theta_{1}\right)$ and $\lambda_{2}=\theta_{1}$; then $\lambda_{1}+$ $\lambda_{2}=1$. Similarly, let $\lambda_{3}=\left(1-\theta_{2}\right)$ and $\lambda_{4}=\theta_{2}$; then $\lambda_{3}+\lambda_{4}=1$. The following expresses the likelihood function in terms of the above models. Consider

$$
\begin{aligned}
P_{c}(j) & =\sum_{\ell=1}^{M} P(\omega=\ell) P\left(\omega_{c}=j \mid \omega=\ell\right) \\
& =\left(\frac{\lambda_{3}}{M}+\lambda_{4}\right) P_{j}+\sum_{\substack{\ell=1 \\
\ell \neq j}}^{M} \frac{\lambda_{3}}{M} P_{\ell}=\frac{\lambda_{3}}{M}+\lambda_{4} P_{j}
\end{aligned}
$$

$$
P_{11}^{\prime}=\frac{\lambda_{1}}{M} \frac{\lambda_{3}}{M}+\left(\lambda_{1} \lambda_{4}+\lambda_{2} \lambda_{3}\right) \frac{P_{1}}{M}+\lambda_{2} \lambda_{4} P_{1}
$$

Similarly, for $i \neq j$,

$$
p_{i j}^{\prime}=\frac{\lambda_{1}}{M} \frac{\lambda_{3}}{M}+\frac{\lambda_{2} \lambda_{3}}{M} P_{i}+\frac{\lambda_{1} \lambda_{4}}{M} P_{j}
$$

Substituting equations (34) through (36) into the likelihood function results in

$$
\begin{aligned}
L= & \log c+\sum_{i=1}^{M} \sum_{\substack{j=1 \\
j \neq 1}}^{M} m_{i j} \log \left(\frac{\lambda_{1} \lambda_{3}}{M^{2}}+\frac{\lambda_{2} \lambda_{3}}{M} P_{i}+\frac{\lambda_{1} \lambda_{4}}{M} p_{j}\right) \\
& +\sum_{i=1}^{M} m_{i j} \log \left[\frac{\lambda_{1} \lambda_{3}}{M^{2}}+\left(\frac{\lambda_{1} \lambda_{4}}{M}+\frac{\lambda_{2} \lambda_{3}}{M}+\lambda_{2} \lambda_{4}\right) p_{i}\right] \\
& +\sum_{i=1}^{M} x_{i} \log \left(\frac{\lambda_{3}}{M}+\lambda_{4} p_{i}\right)
\end{aligned}
$$


Now, the problem can be stated as follows.

$$
\text { Find: } \lambda_{i}(i=1,2,3,4) \text { and } p_{j}(j=1,2, \cdots, M)
$$

so that $L$ of equation (37) is maximized subject to the following constraints.

$$
\left.\begin{array}{l}
\sum_{i=1}^{M} p_{i}=1 \\
\lambda_{1}+\lambda_{2}=1 \\
\lambda_{3}+\lambda_{4}=1 \\
0 ; i=1, \cdots, 4 \\
0 ; i=1,2, \cdots, M
\end{array}\right\}
$$

Optimization techniques, such as the DavidonFletcher-Powell procedure, can be used to maximize L.16-18 The numbers of parameters, equality constraints, and inequality constraints as a function of $M$ are $(4+M), 3$, and $(4+M)$, respectively. It is seen that the optimization problem is considerably simplified.

\section{B. A PRACTICAL APPLICATION}

The maximum likelihood estimation with the simplified models presented in section IIIA is applied to processing remotely sensed Landsat multispectral scanner (MSS) data. Several segments* are processed in the following manner. A linear classifier is trained for two classes. Class 1 is wheat $(W)$ and class 2 is other $(N)$.

*A segment is a 9- by 11-kilometer (5- by 6-nautical mile) area for which the MSS image is divided into a rectangular array of pixels, 117 rows by 196 columns.
This classifier is used to classify a test set of data (104 patterns) for which labels are available and a set of data (209 patterns) for which labels are not available. Thus, the classifications corresponding to table 1 are computed. The labels for the test data are assumed to be imperfect. The maximum likelihood estimates of $\lambda_{i}(i=1,2$, $3,4)$ and $P_{j}(j=1,2)$, subject to the constraints of equation (38), are obtained using the DavidonFletcher-Powell optimization procedure. 16,17

The results obtained from the optimization of the likelihood function are shown in table 2. The last column in table 2 lists the $P(\omega=1)$ values computed from the ground-truth information over the entire segment for each segment. The following conclusions can be made from table 2 . The mean and variance of errors of estimated $P_{1}$ with respect to the ground-truth $\mathrm{P}_{1}$ are smaller with the modeling of imperfections in the labels than with the estimates obtained assuming the labels are perfect. When there are no imperfections in the 1abels (i.e., for segments 1520, 1648, and 1929), the estimates of P $P_{C C}$ 's obtained with and without modeling of imperfections in the labels are identical. Furthermore, when the estimated $P_{c c}$ is 1 (with modeling of label imperfections), the estimated $P_{C c}$ (assuming labels are perfect) is identical with the probability of label imper-

fections. The $P_{1}$ and $P_{1}$ are related as follows:

$$
P_{1}^{\prime}=P\left(\omega^{\prime}=1\right)=\sum_{\ell=1}^{M} P\left(\omega^{\prime}=1 / \omega=\ell\right) P(\omega=\ell)
$$

If it is assumed that the labels are perfect, the estimate of $P_{1}$ is an estimate of $P_{1}$. Table 3 lists the estimate of $\mathrm{P}$ i obtained from equation (39) and that obtained as a maximum likel ihood estimate from equation (10), assuming the labels are perfect.

\begin{tabular}{|c|c|c|c|c|c|c|c|c|}
\hline \multirow{2}{*}{ Segment } & \multicolumn{2}{|c|}{ Site description } & \multicolumn{2}{|c|}{$\begin{array}{l}\text { Without modeling } \\
\text { tmperfections in } \\
\text { the labels }\end{array}$} & \multicolumn{3}{|c|}{$\begin{array}{c}\text { With modeling imperfections } \\
\text { in the labels }\end{array}$} & \multirow{2}{*}{$\begin{array}{c}\text { Ground- } \\
\text { truth } \\
\text { proportion, } \\
P(\omega=1)\end{array}$} \\
\hline & County & State & $P_{1}$ & $P_{c c}^{\prime}$ & $\begin{array}{c}P\left(\omega^{\prime}=1 \mid \omega=1\right) \\
(a)\end{array}$ & ${ }^{P} \mathrm{cc}$ & $\begin{array}{c}P_{1}=P(\omega=1) \\
\text { (b) }\end{array}$ & \\
\hline 1060 & Sherman & Tex. & 0.3421 & 0.8284 & 0.8377 & 0.9905 & 0.2492 & 0.229 \\
\hline 1512 & Clay & Minn. & .4295 & .7653 & .7678 & 1.0000 & .3594 & .337 \\
\hline 1520 & Big Stone & Minn. & .2647 & .7763 & 1.0000 & .7790 & .2759 & .299 \\
\hline 1604 & Renville & N. Dak. & .5506 & .6378 & .7100 & .8363 & .6030 & .526 \\
\hline 1648 & Spink & S. Dak. & 2868 & .8160 & 1.0000 & .8182 & .2894 & .379 \\
\hline 1677 & Spink & S. Dak. & .3838 & .7501 & .7847 & .9445 & .3034 & .341 \\
\hline 1734 & $\mathrm{H}+11$ & Mont. . & .4663 & .8857 & . 8865 & 1.0000 & .4486 & .440 \\
\hline 1929 & Blaine & Mont. & .4445 & .9422 & 1.0000 & .9472 & .4672 & .426 \\
\hline \multicolumn{2}{|c|}{ Mean of errors } & & 0.02391 & & & & 0.002388 & \\
\hline \multicolumn{2}{|c|}{ Variance of errors } & & 0.00374 & & & & 0.002318 & \\
\hline
\end{tabular}

Table 2. Estimates of a Priori Probability and $P_{C c}$ With and Without Modeling of Imperfections in the Labels

AProbablitity of label imperfections.

Estimated proportion of class?. 
Table 3. Comparison of Estimates of Pi With and Without Modeling of Label Imperfections

\begin{tabular}{|c|c|c|}
\hline Segment & $\begin{array}{c}\text { Estimate of } P_{1}^{\prime}, \\
P_{1}^{\prime}=\sum_{j=1}^{n} P\left(\omega^{\prime}=1 \mid \omega=j\right) P(\omega=j)\end{array}$ & $\begin{array}{l}\text { Maxímum likel ihood } \\
\text { estimate of } p_{1}^{\prime} \text { obtained } \\
\text { from equation }(10)\end{array}$ \\
\hline 1060 & 0.3322 & 0.3421 \\
\hline 1512 & .4246 & .4295 \\
\hline 1520 & .2759 & .2647 \\
\hline 1604 & .5432 & .5506 \\
\hline 1648 & .2894 & .2868 \\
\hline 1677 & .3880 & .3838 \\
\hline 1734 & .4602 & .4663 \\
\hline 1929 & .4672 & .4445 \\
\hline
\end{tabular}

Columns 2 and 3 of table 3 are almost identical, thus verifying the validity of the models used in defining the label imperfections.

\section{MAXIMUM LIKELIHOOD ESTIMATION WITH CLASS- DEPENDENT MODELING OF LABEL IMPERFECTIONS AND ERROR PROBABILITIES}

When modeting label imperfections and error probabilities, the $\theta$ 's and hence $\lambda$ 's can be made class dependent, which increases the complexity of the problem. For different $i$ and $j$, the imperfections in the labels can be modeled as

$$
\left.\begin{array}{c}
P\left(\omega^{\prime}=1[\omega=i)=\frac{\left[1-\theta_{1}(i)\right]}{m}+\theta_{1}(i)\right. \\
P\left(\omega^{\prime}=j \mid \omega=1\right)=\frac{\left[1-\theta_{1}(t)\right]}{\pi} \\
0 \leq \theta_{1}(1) \leq 1
\end{array}\right\}
$$

Similarly, for different $i$ and $j$, the error probabilities can be modeled as

$$
\left.\begin{array}{c}
P\left(\omega_{c}=f \mid \omega=i\right)=\frac{\left[1-\theta_{2}(i)\right]}{1}+\theta_{2}(i) \\
P\left(\omega_{c}=j \mid \omega=i\right)=\frac{\left[1-\theta_{2}(i)\right]}{\omega} \\
0 \leq \theta_{2}(t) \leq 1
\end{array}\right\}
$$

It can be shown that these models satisfy the postulates of probability. Let $\lambda_{1}(i)=[1-$ $\left.\theta_{1}(i)\right], \lambda_{2}(i)=\theta_{1}(i), \lambda_{3}(i)=\left[1-\theta_{2}(i)\right]$, and $\lambda_{4}(i)=\theta_{2}(i)$. Then,

$$
\left.\begin{array}{c}
\lambda_{1}(1)+\lambda_{2}(1)=1 ; 1=1,2, \cdots, m \\
\lambda_{3}(1)+\lambda_{4}(1)=1
\end{array}\right\}
$$

An analysis similar to equations (34) through (36) yields the following equations.

$$
P_{c}(j)=\sum_{l=1}^{M} \frac{\lambda_{3}(l)}{N} P_{l}+\lambda_{f}(j) P_{j}
$$

$$
\begin{aligned}
p_{i i}^{\prime}= & \sum_{l=1}^{M} \frac{\lambda_{1}(l)}{M} \frac{\lambda_{3}(l)}{M} P_{l}+\left[\frac{\lambda_{1}(1)}{M} \lambda_{4}(1)+\lambda_{2}(1) \frac{\lambda_{3}(i)}{M}\right. \\
& \left.+\lambda_{2}(1) \lambda_{4}(1)\right] P_{i} \\
P_{i j}^{\prime}= & \sum_{l=1}^{M} \frac{\lambda_{1}(l)}{M} \frac{\lambda_{3}(l)}{M} P_{l}+\left[\lambda_{2}(1) \frac{\lambda_{3}(i)}{M} P_{1}\right. \\
& \left.+\frac{\lambda_{1}(j)}{M} \lambda_{4}(j) P_{j}\right]
\end{aligned}
$$

Equations (43) through (45) can be used to obtain an expression for the likelihood function $L$.

The problem of maximizing the likel ihood function $L$ may be stated as follows:

Find: $\lambda_{1}(j)(j=1,2, \cdots, M ; i=1,2,3,4)$ and $P_{j}(j=1,2, \cdots, M)$

so that $L$ is maximized subject to the following constraints.

$$
\left.\begin{array}{c}
\sum_{i=1}^{M} p_{i}=1 \\
\lambda_{1}(1)+\lambda_{2}(1)=1 ; i=1,2, \cdots, M \\
\lambda_{3}(1)+\lambda_{4}(1)=1 ; i=1,2, \cdots, M
\end{array}\right\}
$$

The optimization technique of Davidon, Fletcher, and Powel116,17 can be used to maximize $L$, subject to the constraints of equations (46) and (47). The numbers of parameters, equality constraints, and inequality constraints as a function of $M$ are $(4 M+M),(2 M+1)$, and $(4 M+M)$, respectively. It is seen that they grow linearly with $M$.

\section{IDENTIFICATION OF MISLABELED PATTERNS}

This section considers the problem of identifying mistabeled patterns, if the probability of Tabel imperfections is either known or estimated using the methods developed in section III. Some relationships are developed between the a priori probabilities and the probability densities with and without imperfections in the labels. The imperfections in the labels are described by the probabilities

$$
B_{j i}=P\left(w^{\prime}=1 / w=j\right) ; 1, j=1,2, \cdots, M
$$

where $i$ and $j$ indicate class. We have the constraint,

$$
\sum_{i=1}^{n} B_{j t}=1
$$


It is assumed that

$$
p(x \mid \omega=j)=p\left(x \mid \omega^{\prime}=1, \omega=j\right)
$$

That is, given the true label of a pattern, the density of the pattern does not depend on its imperfect label. The densities $p(X \mid \omega=i)$ and $p\left(X \mid \omega^{\prime}=i\right)$ are related as

$$
p\left(x \mid \omega^{\prime}=1\right)=\frac{1}{P\left(\omega^{\prime}=1\right)} \sum_{j=1}^{M} \beta_{j j} P(\omega=j) p(x \mid \omega=j)
$$

Similarly, the a priori probabilities are related as

$$
P\left(\omega^{\prime}-i\right)=\sum_{j=1}^{M} \beta_{j 1} P(\omega=j)
$$

Inverting equation (51) yields the following result for the two-class case.

$$
\begin{aligned}
P(\omega=i) p\left(\left.x\right|_{\omega}=i\right)= & \frac{1}{\left(\beta_{i j} \beta_{j j}-\beta_{i j} \beta_{j i}\right.}\left[\beta_{j j} P\left(\omega^{\prime}=i\right)_{p}\left(x \mid \omega^{\prime}=i\right)\right. \\
& \left.-\beta_{j j} P\left(\omega^{\prime}=j\right)_{p}\left(X \mid \omega^{\prime}=j\right)\right] \\
& i, j=1,2 \text { and } i \neq j
\end{aligned}
$$

Let $\beta$ be the matrix of probabilities of label imperfections with the elements $B_{i j}$. That is,

$$
\beta=\left[\beta_{i j}\right]
$$

Assuming $\beta^{-1}$ exists, the following can be obtained from equation (51) in the multiclass case.

$$
\begin{aligned}
P(\omega=1) p\left(\left.x\right|_{\omega}=1\right)= & \sum_{s=1}^{M} \delta_{1 s} P\left(\omega^{\prime}=s\right)_{P}\left(x \mid \omega^{\prime}=s\right) \\
& 1=1,2, \cdots, M
\end{aligned}
$$

A. IDENTIFICATION OF MISLABELED PATTERNS IN THE TWO-CLASS CASE

The following expressions are developed for the identification of mislabeled patterns using a linear classifier. The linear classifier implements a decision criterion

$$
\left.\begin{array}{l}
\text { Decide } x \in \omega^{\prime}=1 \text { if } g(x)=w^{\top} x+w_{0}>0 \\
\text { secide } x \in \omega^{\prime}=2 \text { otherwise }
\end{array}\right\}
$$

It is assumed that $p\left(X \mid \omega^{\prime}=i\right)$ is multivariate normal; i.e., $p\left(\left.X\right|_{\omega} ^{\prime}=i\right) \sim N\left(M_{j}^{\prime}, \Sigma_{j}^{\prime}\right), i=1,2$. Since $g(X)$ is a linear combination of the components of pattern vector $X$, if $X$ is normally distributed, $g(X)$ is also normally distributed. That is,

$$
p\left[g(x) \mid x \in \omega^{\prime}=i\right] \sim N\left[m_{1}^{\prime},\left(\sigma_{1}^{\prime}\right)^{2}\right] ; i=1,2
$$

where

$$
\left.\begin{array}{l}
m_{i}^{\prime}=w^{\top} M_{i}^{\prime}+w_{0} \\
\left(\sigma_{i}^{\prime}\right)^{2}=w^{\top} r_{j} w
\end{array}\right\}
$$

To identify and change the labels of mislabeled patterns, the following scheme is proposed.

$$
\left.\begin{array}{l}
\text { Change the label of } x \text { to } \omega=1 \text { if } g(x)>t_{1} \\
\text { Change the label of } x \text { to } \omega=2 \text { if } g(x)<-t_{2} \\
\text { Do not change the label of } x \text { if }-t_{2} \leq g(x) \leq t_{1}
\end{array}\right\}
$$

The thresholds $t_{1}$ and $-t_{2}$ are used to identify the incorrect labels and are determined by specifying the probability $\alpha$, that mislabeling will occur in the label correction process. An expression for the probability that the Tabel correction scheme will give an incorrect label is derived in the following equation.

$$
\begin{aligned}
P_{B L}= & P(\text { bad label }) \\
= & P(\omega=1) P(\text { bad label } \mid x \in \omega=1) \\
& +P(\omega=2) P(\text { bad label } \mid x \in \omega=2) \\
= & P(\omega=1) P\left[g(x)<-t_{2} \mid x \in \omega=1\right] \\
& +P(\omega=2) P\left[g(x)>t_{1} \mid x \in \omega=2\right]
\end{aligned}
$$

Using equations (53) and (60) obtains the following result.

$$
\begin{aligned}
P(\omega=1) P[g(x) & \left.\leq-t_{2} \mid x \in \omega=1\right] \\
& =\frac{1}{\left(\beta_{11}\right)_{22}^{-\beta_{12} z_{21}}}\left[\beta_{22} P\left(\omega^{\prime}=1\right) \int_{-\infty}^{\frac{-t_{2}-m_{1}^{\prime}}{\sigma_{1}^{\prime}} \psi(y) d y}\right. \\
& \left.-B_{21} P\left(\omega^{\prime}=2\right) \int_{-\infty}^{\frac{-t_{2}-m_{2}^{\prime}}{\sigma_{2}^{\prime}}} \psi(y) d y\right]
\end{aligned}
$$

where

Similarly,

$$
\phi(y)=\frac{1}{\sqrt{2 \pi}} \exp \left(\frac{-y^{2}}{2}\right)
$$

$$
\begin{gathered}
P(\omega=2) P\left[g(x)>t_{1} \mid x \in \omega=2\right]=\frac{1}{\left(\beta_{11} \beta_{22}-\beta_{12} \beta_{21}\right)}\left[\beta_{11} P\left(\omega^{\prime}-2\right)\right. \\
\left.\int_{-\infty}^{\frac{-t_{1}+\pi_{2}^{\prime}}{\sigma_{2}^{\prime}}} \psi(y) d y-\beta_{12} P\left(\omega^{\prime}=1\right) \int_{-\infty}^{\frac{-t_{1}+m_{1}^{\prime}}{\sigma_{1}^{\prime}}} \psi(y) d y\right]
\end{gathered}
$$

Equations (60) through (63) yield an expression for the probability of bad label $P_{B L}$.

For a given $\alpha, t_{1}$ and $-t_{2}$ can be computed using an optimization technique such as the Davidon-Fletcher-Powell procedure, so that the square of the error between $\alpha$ and $\mathrm{PBL}_{\mathrm{BL}}$ is minimized and can be used in the incorrect label identification scheme. 
B. AN EXAMPLE OF APPLICATION OF THE INCORRECT LABEL IDENTIFICATION SCHEME

The two-class imperfect label correction scheme presented in section IVA is applied to a practical problem in remote sensing. In particular, it is applied to Landsat imagery of segment 1060. Data from two acquisitions are processed, and each acquisition has four spectral bands. The image is overlaid with a rectangular grid of 209 grid intersections, and the labels of pixels corresponding to each grid intersection are acquired. A linear classifier is trained on onehalf of the data. The remaining one-half of the data is used as a test data set. Test data set and total data set classifications are obtained using the linear classifier. This results in matrices corresponding to table $1(a)$ and $(b)$. The maximum likelihood estimates of label imperfections are obtained using the simplified models presented in section IIIA. The $\beta$-matrix and the a priori probabilities obtained are

$$
\left.\begin{array}{l}
\beta=\left[\begin{array}{ll}
0.8378 & 0.1622 \\
0.1622 & 0.8378
\end{array}\right] \\
P(\omega=1)=0.24921 \\
P(\omega=2)=0.75079
\end{array}\right\}
$$

Choosing $\alpha=0.001$, upper and lower thresholds $t_{1}$ and $-t_{2}$ that minimize the square of the difference between $\alpha$ and $P_{B L}$ are computed using the Davidon-Fletcher-Powe 11 procedure. The patterns of class $\omega^{\prime}=2$, the discriminant function values of which exceeded $t_{7}$, and the patterns of class $\omega^{\prime}=1$, the discriminant function values of which are less than $-t_{2}$, are identified and marked with circles in figures 1 and 2 . These figures list the labels of the pixels of 209 grid intersections and their relative positions.

Films of the two acquisitions of segment 1060 used in the processing were examined by an analyst-interpreter (AI), and the results are given in figures 3 and 4 .

From an analysis of figures 3 and 4 , it can be concluded that the decisions of the label correction scheme are in close agreement with the AI interpretations of the imagery films.

C. IDENTIFICATION OF MISLABELED PATTERNS IN THE MULTICLASS CASE

Let $g_{j}(x)$ be the discriminant function of the ith class $\omega^{\prime}=i$, where

$$
g_{i}(x)=w_{i}^{\top} x+w_{10} ; i=1,2, \cdots, M
$$

The usual decision criterion in a multiclass

\begin{tabular}{|c|c|c|c|c|c|c|c|c|c|c|c|c|c|c|c|c|c|c|c|}
\hline & 1 & 2 & 3 & 4 & 5 & . & & 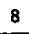 & & 10 & $n$ & 12 & 13 & 14 & 15 & 16 & 17 & 18 & \\
\hline 1 & $N$ & (i) & $\mathrm{N}$ & N & N & $\mathbf{N}$ & " & $\mathrm{N}$ & N & $\mathrm{N}$ & $\mathbf{N}$ & & N & & & N & N & N & N \\
\hline 2 & N & $\mathrm{N}$ & $N$ & N & & & 1 & $\mathrm{~N}$ & $\mathbf{N}$ & N & 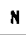 & & & & $N$ & N & $N$ & $\mathrm{H}$ & \\
\hline 3 & $\mathrm{~N}$ & $N$ & N & N & N & & N & & & $N$ & & N & & $\mathrm{N}$ & $\left(\mathbb{Q}_{\mathrm{B}}\right.$ & N & & & \\
\hline 4 & $N$ & N & $N$ & H & "I & & N & & & N & M & N & $\mathrm{N}$ & $\mathbf{N}$ & & (1) & & & \\
\hline 5 & N & $N$ & N & $\mathbf{N}$ & N & & & & & & N & N & $N$ & $N$ & $N$ & $N$ & N & (1) & \\
\hline 6 & N & N & N & N & $n$ & & & & & & & $M$ & K & N & & & $\boldsymbol{H}$ & N & \\
\hline 7 & $\mathrm{~N}$ & N & & N & N & & N & & & & H & N & $\mathbf{N}$ & M & N & N & & N & \\
\hline 8 & N & & N & N & $n$ & & & & $\mathbf{N}$ & & & N & $\mathrm{N}$ & $\mathbf{N}$ & N & & N & $\mathrm{N}$ & N \\
\hline 9 & $N$ & & & N & & $\mathrm{N}$ & $\mathrm{N}$ & & N & N & 1 & & & & & & & N & \\
\hline 10 & & N & N & N & & & $\mathrm{N}$ & $\mathbf{N}$ & & & (ii) & & & & $N$ & " & & N & \\
\hline & & & & & (1) & & $\mathrm{N}$ & & & & $\mathbf{N}$ & & & $\mathrm{N}$ & $\mathrm{N}$ & $1 \mathrm{~N}$ & 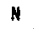 & N & \\
\hline
\end{tabular}
case is to decide $X \in \omega^{\prime}=l$, if

$$
g_{\ell}(x)=\max _{\substack{j=1 \\ j \neq \ell}} g_{j}(x), \cdots, M
$$

Computed upper threshold $t_{1}=0.1507$

\begin{tabular}{|c|c|c|c|c|c|c|c|c|c|c|c|c|c|c|c|c|c|c|c|}
\hline & 1 & 2 & 3 & 4 & S & 6 & 7 & 8 & 9 & 10 & II & 12 & 13 & 14 & 15 & 16 & 17 & 18 & 19 \\
\hline 1 & & & & & & & & & & & & $w$ & & (W) & $w$ & & & & \\
\hline 2 & & & & & (4) & (14) & & & & & & (4) & (10) & (4) & & & & & \\
\hline 3 & & & & & & & & & & & & & $W$ & & & & W & (1) & (1) \\
\hline 4 & & & & & & W & & & $w$ & & & & & & (1) & & $n$ & $W$ & H \\
\hline 5 & & & & & & $w$ & (4) & $w$ & $w$ & W & & & & & & & & & $H$ \\
\hline 6 & & & & & & & (1) & $w$ & $w$ & $w$ & $W$ & & & & $W$ & (4) & & & \\
\hline 7 & & & (1) & & & & & $W$ & (ㄴ) & & & & & & & & (1) & & \\
\hline 8 & & (1) & & & & $N$ & $H$ & $w$ & & W & N & & & & & (1) & & & \\
\hline 9 & & (W) & $w$ & & $n$ & & & & & & & $w$ & $\omega$ & $W$ & (1) & $H$ & $w$ & & (1) \\
\hline 10 & (1) & & & & $W$ & $w$ & & & $w$ & $W$ & & $w$ & $w$ & $W$ & & & (i) & & \\
\hline 11 & $N$ & W & $\left(\mathbb{W}^{*}\right.$ & $w$ & & $W$ & & & & & & $H$ & & & & & & & \\
\hline
\end{tabular}

\begin{tabular}{|l|l|}
\hline \multicolumn{2}{|c|}{ Legend } \\
\hline Blank & Wheat pixels \\
N & Other pixels \\
(i) & Pixels identified by label correction scheme as wheat \\
B & AI decision as wheat but bordering class other \\
* & Al decision as other \\
\hline
\end{tabular}

Figure 1. Diagram of 209 Grid Intersections Showing Pixels Labeled Other and Other Pixels Reidentified as Wheat Using Imperfect Label Ident ification Scheme.

Computed lower threshold $-t_{2}=-0.01628$

\begin{tabular}{|l|l|}
\hline \multicolumn{2}{|c|}{ Legend } \\
\hline Blank & Other pixels \\
W & Wheat pixels \\
B & Pixels identified by label correction scheme as other \\
& AI decision as other but bordering wheat \\
\hline
\end{tabular}

Figure 2. Diagram of 209 Grid Intersections Showing Pixels Labeled Wheat and Wheat Pixels Identified as Other Using Imperfect Label Identification Scheme. 


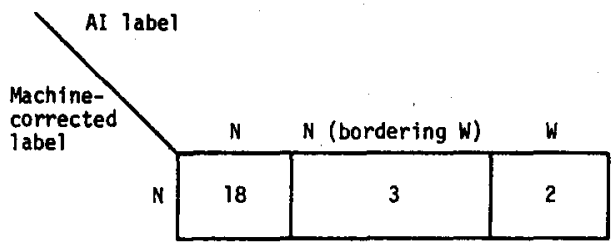

Figure 3. AI Labels for Patterns Where Labels Were Changed From Wheat to Other.

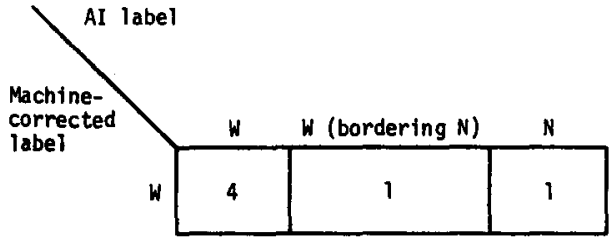

Figure 4. AI Labels for Patterns Where Labels Were Changed From Other to Wheat.

To identify and change the labels of mislabeled patterns, the following scheme is proposed:

Change the label of $x$ from $\omega^{\prime}=\mathbf{i}$ to $\omega=\ell$ if

$$
g_{\ell}(x)=\max _{\substack{j=1 \\ j \neq i}} g_{j}(x)>g_{i}(x)+t_{j}
$$

where $t_{i}$ is a positive number.

Otherwise, do not change the label of $x$.

The threshold $t_{j}$ for identifying the incorrect labels is determined by specifying the probability $\alpha$, that mislabeling will occur in the label correction process of equation (68). An upper bound on the probability that such a scheme gives an incorrect label is derived as follows.

$$
\begin{aligned}
P_{B L} & =\sum_{j=1}^{M} P(\omega=i) P\left[g_{\ell}(x)=\max _{\substack{j \\
j=1,2, \cdots, M \\
j \neq i}} g_{j}(x)>g_{i}(x)+t_{j} \mid \omega=i\right] \\
& \leq \sum_{\substack { j=1 \\
\begin{subarray}{c}{j=1 \\
j \neq i{ j = 1 \\
\begin{subarray} { c } { j = 1 \\
j \neq i } }\end{subarray}}^{M} P(\omega=1) P\left[g_{j}(x)>g_{j}(x)+t_{i} \mid \omega=i\right]
\end{aligned}
$$

It is assumed that the densities $p\left(X \mid \omega^{\prime}=i\right)$ are multivariate normal. That is, $p\left(X \mid \omega^{\prime}=i\right) \sim$ $N\left(M_{j}^{\prime}, \Sigma i j\right), i=1,2, \cdots, M$.

$$
\text { Let } \quad \begin{aligned}
g_{j i}(x) & =g_{j}(x)-g_{i}(x) \\
& =w_{j i}^{\top} x+w_{j 10}
\end{aligned}
$$

Since $g_{j j}(x)$ is a linear combination of the components of pattern vector $X$, if $X$ is normally distributed, $g_{j i}(X)$ is also normally distributed. That is,

$$
P\left[g_{j i}(x) \mid \omega^{\prime}=s\right] \sim N\left[m_{j 1 s}^{\prime},\left(\sigma_{j i s}^{\prime}\right)^{2}\right]
$$

where

$$
\left.\begin{array}{l}
m_{j i s}^{\prime}=w_{j i}^{\top} M_{s}^{\prime}+w_{j i 0} \\
\left(\sigma_{j i s}^{\prime}\right)^{2}=w_{j i}^{\top} \Sigma_{s}^{\prime} w_{j i}
\end{array}\right\}
$$

From equations (55), (69), and (72), the following is obtained.

$$
\begin{aligned}
P_{B L} & \leq \sum_{i=1}^{M} \sum_{\substack{j=1 \\
j \neq 1}}^{M} \sum_{s=1}^{M} \delta_{i s} P\left(w^{\prime}=s\right) P\left[g_{j}(x)>g_{i}(x)+t_{i} \mid w^{\prime}=s\right] \\
& =\sum_{i=1}^{M} \sum_{\substack{j=1 \\
j \neq 1}}^{M} \sum_{s=1}^{M} \delta_{i s} P\left(w^{\prime}=s\right) \int_{-\infty}^{-t_{j}+m_{j i s}^{\prime}} \frac{\sigma_{j i s}^{\prime}}{d y} d y
\end{aligned}
$$

where $\psi(y)$ is given by equation (62). The thresholds $t_{j}(i=1,2, \cdots, M)$ can be determined using an optimization technique such as the Davidon-Fletcher-Powell procedure. However, it is to be noted that when $M=2$, equations $(60)$ and (73) are identical. The imperfect label identification scheme in the multiclass case amounts to establishing a region around each decision surface.

\section{CONCLUSIONS}

In the practical applications of pattern recognition, obtaining labels for the patterns is expensive and very often these labels are imperfect. This paper has presented the problem of estimating imperfections in the labels and the use of these estimates in the identification of mislabeled patterns.

It is assumed that a set of labeled patterns, the labels of which might be imperfect, and a set of unlabeled patterns are available. The classifier classifies these patterns, and the results are a confusion matrix for the labeled pattern set and classification counts for the unlabeled set.

Expressions are presented for the maximum likelihood estimates of classification errors, for percentages of correct classification and proportions, and for the asymptotic variances of probability of correct classification and proportions.

Assuming imperfections in the labels, simple models are presented for modeling imperfections in the labels and classification errors. The problem of maximum likelihood estimation of various quantities is formulated for a general case, in terms of simplified models and class-dependent models. and their relative complexities are discussed. Results of practical applications of maximum likelihood estimation of various quantitiés are presented.

Assuming the densities are Gaussian and the probabilities of label imperfections are known, thresholding schemes are proposed for the identification of mislabeled patterns both for the twoclass and the multiclass cases. The probability 
that such an identification scheme results in a wrong decision for a pattern is expressed as a function of the thresholds, and the thresholds can be computed by specifying the probability of a wrong decision by the imperfect label identification scheme.

Furthermore, the results of applying these techniques to the processing of remotely sensed multispectral data are presented.

\section{APPENDIX A}

MAXIMUM LIKELIHOOD ESTIMATION THROUGH MODELING OF

$$
P\left(\omega^{\prime}=i \mid \omega=\ell, \omega_{C}=j\right)
$$

In section III, an assumption is made [equation (22)] that, given the true label and the classifier label, the imperfect label depends only on the true label. In this appendix, the quantity in the right-hand side of equation (23) is modeled and is used in formulating the maximum likelihood estimation scheme. The information in the classifier label $\omega_{c}=j$, about the imperfect label $\omega^{\prime}=i$, can be modeled as follows.

$$
\begin{aligned}
& P\left(\omega^{\prime}=j \mid \omega=\ell, \omega_{c}=j\right)=\frac{P\left(\omega^{\prime}=j \mid \omega=\ell\right)}{(1-\theta)+\theta P\left(\omega^{\prime}=j(\omega=\ell)\right.} \\
& P\left(\omega^{\prime}=1 \omega_{\omega}=2, \omega_{c}=j\right)=\frac{(1-\theta) P\left(\omega^{\prime}=1 / \omega=l\right)}{(1-\theta)+\theta P\left(\omega^{\prime}=j(\omega)=\ell\right)} ; 1 \neq j,
\end{aligned}
$$

where $\theta<1$. The justification for the models of equations (74) and (75) is as follows. By using the Bayesian rule, the left-hand side of equation (75) can be written as

$$
P\left(\omega^{\prime}=i \mid \omega=\ell, \omega_{c}=j\right)=\frac{P\left(\omega^{+}=i \omega_{c}=j \mid \omega=\ell\right)}{P\left(\omega_{c}=j \mid \omega=\ell\right)}
$$

Consider the numerator of equation (76). It can be modeled through the following.

1. If the classifier and imperfect labeler are conditionally independent, for different $i$ and $j$,

$$
\left.\begin{array}{l}
P\left(\omega^{\prime}=i, \omega \omega_{c}=i \mid \omega=l\right)=P\left(\omega^{\prime}=i \mid \omega=l\right) P\left(\omega_{c}=i \mid \omega=l\right) \\
P\left(\omega^{\prime}=i, \omega_{c}=j(\omega=l)=P\left(\omega^{\prime}=i \mid \omega=l\right) P\left(\omega_{c}=j \mid \omega=l\right)\right.
\end{array}\right\}
$$

2. If the classifier and imperfect labeler are in complete agreement, for different $i$ and $j$,

$$
\left.\begin{array}{rl}
P\left(\omega^{\prime}=1, \omega_{c}\right. & =i \mid \omega=\ell)=P\left(\omega_{c}=1 \mid \omega=\ell\right) \\
P\left(\omega^{\prime}\right. & \left.=i, \omega_{c}=j \mid \omega=\ell\right)=0
\end{array}\right\}
$$

Since, in general, the truth lies somewhere between the above two extremes, using a parameter $\theta$, one can write for different $i$ and $j$

$$
\left.\begin{array}{c}
P\left(\omega^{\prime}=j, \omega_{c}=j \mid \omega=\ell\right)=\frac{P\left(\omega_{c}=j \mid \omega=\ell\right) P\left(\omega^{\prime}=j \mid \omega=\ell\right)}{(T-\theta)+\theta P\left(\omega^{\prime}=j \mid \omega^{\prime}=\ell\right)} \\
P\left(\omega^{\prime}=1, \omega_{c}=j \mid \omega=\ell\right)=\frac{(1-\theta) P\left(\omega_{c}=j \mid \omega=\ell\right) P\left(\omega^{\prime}=1 \mid \omega=\ell\right)}{(1-\theta)+\theta P\left(\omega^{\prime}=j \mid \omega=l\right)}
\end{array}\right\}
$$

From equations (79) and (76), equations and (75) are obtained. Instead of nonlinear interpolation between the extremes of equations (77) and (78), use of linear interpolation results in the following models.

$$
\left.\begin{array}{r}
P\left(\omega^{\prime}=j \mid \omega=\ell, \omega_{c}=j\right)=(1-\theta) P\left(\omega^{\prime}=j \mid \omega=l\right)+\theta \\
P\left(\omega^{\prime}=1 \mid \omega=\ell, \omega c=j\right)=(1-\theta) P\left(\omega^{\prime}=i \mid \omega=\ell\right)
\end{array}\right\}
$$

For the models of equations (74), (75), and (80), it can be shown that

$$
\sum_{j=1}^{M} P\left(\omega^{\prime}=i \mid \omega=\ell, \omega_{c}=j\right)=1
$$

thus satisfying the probability rule. The models of equations (74) and (75) are used in the rest of this appendix.

From equations (29); (33), (74), and (75) $p_{i j}^{\prime}$ 's can be written as follows.

$$
\begin{aligned}
p_{i j}^{\prime}= & \frac{\frac{\lambda_{1}}{M} \frac{\lambda_{3}}{M}}{(1-\theta)+\theta \frac{\lambda_{1}}{M}}+\frac{P_{i}}{(1-\theta)+\theta\left(\frac{\lambda_{1}}{M}+\lambda_{2}\right)} \\
& \times\left[\frac{\lambda_{1}}{M} \lambda_{4}+\lambda_{2}\left(\frac{\lambda_{3}}{M}+\lambda_{4}\right)-\frac{\frac{\lambda_{1}}{M} \frac{\lambda_{3}}{M} \lambda_{2} \theta}{(1-\theta)+\theta \frac{\lambda_{1}}{M}}\right] \\
p_{i j}^{\prime}= & \frac{(1-\theta) \frac{\lambda_{3}}{M}}{(1-\theta)+\theta \frac{\lambda_{1}}{M}}\left(\frac{\lambda_{1}}{M}+\lambda_{2} p_{1}\right) \\
& +\frac{(1-\theta) \frac{\lambda_{1}}{M} P_{j}}{(1-\theta)+\theta\left(\frac{\lambda_{1}}{M}+\lambda_{2}\right)}\left[\lambda_{4}-\frac{\theta \lambda_{2} \frac{\lambda_{3}}{M}}{(1-\theta)+\theta \frac{\lambda_{7}}{M}}\right]
\end{aligned}
$$

Using equations (34), (82), and (83) in equation (25), an expression for the likelihood function can be obtained. On comparing to the formulation of section IIIA, in this case, it is easily seen that the numbers of parameters and inequality constraints are increased by 1 .

\section{APPENDIX B}

AN ESTIMATION SCHEME WITH THE DEPENDENCIES BETWEEN $P\left(\omega^{\prime}=i \mid \omega=\ell\right)$ AND $P\left(\omega_{c}=\left.j\right|_{\omega}=\ell\right)$

In the maximization of the likelihood function $L$ of equation (25), in section III, it is implicitly assumed that the probabilities $P\left(\omega^{\prime}=i \mid \omega=\ell\right)$ and $P\left(\omega_{c}=j \mid \omega=\ell\right)$ are independent. In general, the sequence of occurrence of different labels is as shown below.

$\underset{\substack{\text { True } \\ \text { label }}}{\substack{\text { Imperfect } \\ \text { label } \\ \omega^{\prime}}} \longrightarrow \underset{\begin{array}{c}\text { Classifter } \\ \text { label } \\ \omega_{c}\end{array}}{\longrightarrow}$

Constraints that can be used with the maximum likelihood estimation schemes of section III, 
relating the probabilities $P\left(\omega^{\prime}=i \mid \omega=\ell\right)$ and $P\left(\omega_{c}=j \mid \omega=\ell\right)$, can be derived as follows. Consider

$$
\begin{aligned}
P\left(\omega_{c}=j \mid \omega=\ell\right) & =\sum_{k=1}^{M} P\left(\omega_{c}=j, \omega^{\prime}=k \mid \omega=2\right) \\
& =\sum_{k=1}^{M} P\left(\omega_{c}=j \mid \omega^{\prime}=k, \omega=\ell\right) P\left(\omega^{\prime}=k(\omega=\ell)^{\prime}\right.
\end{aligned}
$$

The first term in the summation of equation (84) can be modeled as in appendix A. That is,

$$
\left.\begin{array}{c}
P\left(\omega_{c}=j \mid \omega^{\prime}=k, \infty=l\right)=\frac{(1-\theta) P\left(\omega_{c}=j \omega_{\omega}=l\right)}{(1-\theta)+\theta P\left(\omega_{c}=k \omega_{\infty}=l\right)} ; j * k \\
P\left(\omega_{c}=k\left(\omega^{\prime}=k, \infty=l\right)=\frac{P\left(\omega_{c}=k \mid \omega=l\right)}{(1-\theta)+\theta P\left(\omega_{c}=k \mid \omega-l\right)}\right. \\
-\leq \leq 0 \leq 1
\end{array}\right\}
$$

Therefore, the constraints become

$$
\begin{aligned}
& P\left(\omega_{c}=j \mid \omega-\ell\right)=\frac{P\left(\omega_{c}-j \mid \omega-\ell\right) P\left(\omega^{\prime}-j \mid \omega-\ell\right)}{(1-\theta)+\theta P\left(\omega_{c}-j \omega_{\omega}=\ell\right)} \\
& +\sum_{\substack{k=j \\
k \neq j}}^{M} \frac{(1-\theta) P\left(w_{c}=j w_{0}=2\right) P\left(w^{\prime}=k(w-2)\right.}{(1-\theta)+\theta P\left(w_{c}=k p=l\right)}
\end{aligned}
$$

for $j, l=1,2, \cdots, M$.

The constraints of equation (86), together with the maximum likelihood estimation schemes of section III, can be used for the estimation of various probabilities. Also, the maximum likelihood estimation scheme can be reformulated as follows. Consider

$$
\begin{gathered}
P_{i j}^{\prime}=P\left(\omega^{\prime}=1, \omega_{c}=j\right)=P\left(\omega^{\prime}=i\right) P\left(\omega_{c}=j \mid \omega^{\prime}=i\right) \\
P_{c}(j)=\sum_{\ell=1}^{M} P\left(\omega_{c}=j \mid \omega^{\prime}=\ell\right) P\left(\omega^{\prime}=\ell\right)
\end{gathered}
$$

and

$$
P\left(w^{\prime}=\ell\right)=\sum_{r=1}^{M} P\left(w^{\circ}=\ell \mid \omega=r\right) P(\omega=r)
$$

Now the likelihood function can be maximized to solve for the variables $P\left(\omega_{c}=j \mid \omega^{\prime}=i\right)$, $P\left(\omega^{\prime}=\ell \mid \omega=r\right)$, and $P(\omega=r)$ through the various models discussed in the paper:

\section{REFERENCES}

1. Duda, R. 0.; and Singleton, R. C.: Training a Threshold Logic Unit With Imperfectly Classified Patterns. Presented at the WESCON Conv. (Los Angeles), Aug. 1964.

2. Kashyap, R. L.: Algorithms for Pattern Classification. Adaptive, Learning, and Pattern Recognition. Systems, Academic Press (New York), 1970, pp. 81-113.

3. Shanmugam, K.; and Breiphol, A. M.: An Error Correcting Procedure for Learning With an Imperfect Teacher: IEEE Trans. Systems, Man and Cybernetics, July 1971, pp. 223-229.

4. Chittineni, C. B.: Learning With Imperfectly Labeled Patterns. Lockheed Electronics Co., Inc., Technical Memorandum LEC-13068,
JSC-14867, NASA/JSC (Houston), Apr. 1979; Proc. 1979 IEEE Conf. on Pattern Recognition and Image Processing (Chicago), Aug. 6-9, 1979, pp. 52-62.

5. Chittineni, C. B.: On the Selection of Effective Features From the Imperfectly Labeled Patterns. Int. J. of Computer and Information Sciences, vol. 2, no. 2, 1973, pp. 103-114.

6. Chittineni, C. B.: On Feature Extraction From Imperfectly Labeled Patterns. IEEE Trans. Systems, Man and Cybernetics, May 1973, pp. 290-292.

7. Chittineni, C. B.: On the Application of Probabilistic Distance Measures for the Extraction of Features From Imperfectly Labeled Patterns. Proc. 6th Annual Princeton Conf. on Information Sciences and Systems, Mar. 1972.

8. Highleyman, H. H.: The Design and Analys is of Pattern Recognition Experiments. Bell System Tech. J., vol. 41, 1962, pp. 723-744.

9. Fukunaga, K.; and Kessel1, D. L.: Nonparametric Bayes Error Estimation Using Unclassified Samples. IEEE Trans. on Information Theory, vol. IT-19, 1973, pp. 434-440.

10. Havens, K. A.; Minter, T. C.; and Thadani, S. G.: Estimation of the Probability of Error Without Ground Truth and Known a Priori Probabilities. IEEE Trans. on Geoscience Electronics, vol. GE-15, no. 3, July 1977 , pp. 147-152.

11. Chow, C. K.: On Optimum Recognition Error and Reject Tradeoff. IEEE Trans. on Information Theory, vol. IT-16, Jan. 1970, pp. 41-46.

12. Chittineni, C. B.: On the Estimation of Probability of Error. Pattern Recognition, vol. 9, no. 4, 1977, pp. 197-196.

13. Chittineni, C. B.: Fisher Classifier and Its Probability of Error Estimation. LEC-13301 (JSC-14865), May 1979.

14. Rao, C. R.: Linear Statistical Inference and Its Applications. John Wiley and Sons, Inc. (New York), 1965.

15. Tenenbein, A.: Estimation From Data Subject to Measurement Error. Ph. D. dissertation, Statistics Department, Harvard University (Cambridge, Mass.), 1969.

16. Fletcher, R.; and Powell, M. J. D.: A Rapidly Convergent Descent Method for Minimi zation. Computer J., vol. 6, Apr. 1963, pp. 163-168.

17. Davidon, W. C.: Variable Metric Method for Minimization. Atomic Energy Commission Research and Development Report ANL-5990 (rev.), Argonne National Laboratory (Lemont, I11.), Nov. 1959.

18. Cooper, L.; and Steinberg, D.: Introduction to Methods of Optimization. W. B. Saunders Co. (Philadelphia), 1970. 